H. ISHIDA

KODAI MATH. J.

16 (1993), 253-268

\title{
NORMAL OPERATORS CONSTRUCTED FROM GENERALIZED HARMONIC MEASURES ON OPEN RIEMANN SURFACES
}

Dedicated to Professor Nobuyuki Suita on his 60th birthday

\author{
BY HISASHI ISHIDA
}

\section{Introduction.}

Let $R$ be an open Riemann surface and $V$ be a union of a finite number of regular subregions in $R$ with disjoint closures. We assume that $R-\bar{V}$ is connected. Denote by $C^{\omega}(\partial V)$ the space of real analytic functions on $\partial V$ and by $H(R-V)$ the space of harmonic functions on $R-V$. A linear operator $L$ from $C^{\omega}(\partial V)$ to $H(R-V)$ is called a normal operator if $L$ satisfies the following conditions:

$$
\begin{gathered}
\left.L f\right|_{\partial V}=f, \\
\min _{\partial V} f \leqq L f \leqq \max _{\partial V} f, \\
\int_{\partial V} * d L f=0 .
\end{gathered}
$$

The notion of normal operators was introduced by L. Sario [13]. He constructed two normal operators $L_{0}$ and $L_{1}$. Here we are specially concerned with $L_{1^{-}}$ operator. If $R$ is a compact bordered surface with smooth boundary, $L_{1} f$ is characterized by the following additional properties:

$$
\begin{gathered}
L_{1} f=\text { constant on } \beta_{\jmath}, \\
\int_{\beta_{3}} * d L_{1} f=0,
\end{gathered}
$$

where $\beta$, are the boundary components of $R$. For a general open Riemann surface $R, L_{1} f$ is defined as $\lim _{n \rightarrow \infty} L_{1}^{R} f f$, where $\left\{R_{n}\right\}$ is a canonical exhaustion of $R$ and $L_{1}^{R_{n}}$ is the $L_{1}$-operator from $C^{\omega}(\partial V)$ to $H\left(R_{n}-V\right)$.

Let $\Gamma_{h}(R)$ be the Hilbert space of real square integrable harmonic differentials on $R$ and $\Gamma_{h s e}(R)$ be the space of semiexact differentials in $\Gamma_{h}(R)$. Let us denote by $\Gamma_{h m}(R)$ the orthogonal complement of $* \Gamma_{h s e}(R)$ in $L_{h}(R)$. Then $L_{1} f$

Received October 14, 1992. 
is characterized by the following properties:

There exist a harmonic function $u_{h m}$ on $R$ with $d u_{h m} \in \Gamma_{h m}(R)$ and a Dirichlet potential $p$ on $R$ such that

on $R-V$ and

$$
L_{1} f=u_{h m}+p
$$

$$
\int_{c} * d L_{1} f=0
$$

for all dividing cycles $c=\partial \Omega$ with $\Omega \subset R-V$.

In [7], we introduced $\Gamma \widehat{h m}$ the space generated by the differentials of generalized harmonic measures and $\Gamma_{h w e}$ the space of harmonic differentials which have vanishing periods along almost all weakly dividing cycles.

In the present paper we construct a normal operator $\hat{L}_{1}$, which is a generalization of $L_{1}$-operator. In contrast with $L_{1} f, \hat{L}_{1} f$ is characterized by the following properties:

There exist a harmonic function $u_{\hat{h m}}$ on $R$ with $d u_{h m} \in \Gamma \widehat{h m}(R)$ and a Dirichlet potential $p$ on $R$ such that

on $R-V$ and

$$
\hat{L}_{1} f=u_{\hat{h m}}+p
$$

$$
\int_{\hat{c}} * d \hat{L}_{1} f=0
$$

for almost all weakly dividing cycles $\hat{c}=\partial G$ with $G \subset R-V$.

Roughly speaking $\hat{L}_{1} f$ takes a constant value on each connected component of the Royden harmonic boundary of $R$ and $* d \hat{L}_{1} f$ has vanishing period along cycles dividing the components of the Royden harmonic boundary.

First, we shall define a finite partition $(P)$ of the Royden harmonic boundary and define the subspaces $(P) \Gamma_{h m}(R)$ and $(P) \Gamma_{h w e}(R)$ of $\Gamma_{h}(R)$. Further we shall define periods of a differential along components of the harmonic boundary.

Next, we construct $(P) \hat{L}_{1}$-operator and $\hat{L}_{1}$-operator. We also study an extremal property of $\hat{L}_{1}$-operator.

Finally, we shall introduce a modulus function obtained from $\hat{L}_{1}$-operator and give an example related to the topic.

\section{Preliminaries.}

Let $R$ be an open Riemann surface and $\Gamma=\Gamma(R)$ the Hilbert space of real square integrable differentials on $R$ (cf. [2]). For $\omega_{1}, \omega_{2} \in \Gamma(R),\left(\omega_{1}, \omega_{2}\right)_{R}=$ $\int_{R} \omega_{1} \wedge * \omega_{2}$ denotes the inner product of $\omega_{1}, \omega_{2}$. where $* \omega$ is the conjugate differential of $\omega$ and $\|\omega\|_{R}$ denotes the norm of $\omega$ on $R$.

We use the notation $|\omega|$ for the density $\sqrt{a^{2}+b^{2}}|d z|$ if $\omega=a d x+b d y$ locally. For the sake of convenience we recall some definitions of subspaces of $\Gamma$ used below. Let $\Gamma_{e}$ be the space of exact differentials in $\Gamma$ and $\Gamma_{e 0}$ be the closure 
NORMAL OPERATORS CONSTRUCTED FROM GENERALIZED HARMONIC MEASURES 255

of differentials of $C^{1}$-functions with compact supports. Let $\Gamma_{h}$ the space of harmonic differentials in $\Gamma, \Gamma_{h s e}$ be the space of semiexact differentials in $\Gamma_{h}$ and $\Gamma_{h e}=\Gamma_{h} \cap \Gamma_{e}$. We denote by $\Gamma_{h m}$ the orthogonal complement of $* \Gamma_{h s e}$ in $\Gamma_{h}$, where $* \Gamma_{x}$ is the class of differentials conjugate to those in $\Gamma_{x}$. Then the following orthogonal decompositions are well known:

$$
\begin{gathered}
\Gamma=\Gamma_{h}+\Gamma_{e 0}+{ }^{*} \Gamma_{e 0}, \\
\Gamma_{e}=\Gamma_{h e}+\Gamma_{e 0} .
\end{gathered}
$$

Let $D(R)$ be the class of real continuous Dirichlet functions on $R$ and $B D(R)$ be the class of bounded functions in $D(R)$ (cf. [3], [14]). Let $H D(R)$ (resp. $H B D(R)$ ) be the class of harmonic functions in $D(R)$ (resp. $B D(R)$ ) and $D_{0}(R)$ (resp. $B D_{0}(R)$ ) be the class of potentials in $D(R)$ (resp. $B D(R)$ ). Since $d D_{0}=\left\{d f ; f \in D_{0}\right\} \subset \Gamma_{e 0}$, we have $(\sigma, d p)_{R}=0$ for any $\sigma \in \Gamma_{h}(R)$ and $p \in D_{0}(R)$. The class $B D(R)$ forms an algebra and the class $D(R)$ has the following lattice property ; if $f, g \in D(R)$ then $f \cup g=\max (f, g)$ and $f \cap g=\min (f, g)$ belong to $D(R)$.

Let $R^{*}$ be the Royden compactification of $R$ and $\Delta$ the (Royden) harmonic boundary of $R$. Every function $f$ in $D(R)$ can be extended continuously to $R^{*}$. Since the extension of $f$ is unique, we may use the same notation $f$ for the extension.

We know that $B D(R)$ enjoys the following Urysohn's property. That is, for any two non-empty disjoint compact sets $K_{1}, K_{2}$ in $R^{*}$ and two real values $a_{1}, a_{2}$, there is a function $f$ in $B D(R)$ such that $f=a_{\imath}$ on $K_{2}(i=1,2)$ and $\min \left(a_{1}, a_{2}\right) \leqq f \leqq \max \left(a_{1}, a_{2}\right)$.

We use the following lemmas ([14]) in the sequal.

LEMMA 1.1. Let $\left\{f_{n}\right\}$ be a sequence of functions in $B D_{0}(R)$ and $f$ a bounded function on $R$. If $\left\|d f_{n}\right\|_{R}$ is uniformly bounded and $\left\{f_{n}\right\}$ converges to $f$ uniformly on every compact subset of $R$ then $f \in B D_{0}(R)$.

Lemma 1.2. A BD-function (resp. D-function) $f$ on $R$ belongs to $B D_{0}(R)$ (resp. $\left.D_{0}(R)\right)$ if and only if $f=0$ on $\Delta$.

LeMma 1.3. Any BD-function (resp. D-function) $f$ on $R$ can be uniquely decomposed into the form $f=u+p$, where $u \in H B D(R)$ (resp. $H D(R)$ ) and $p \in$ $B D_{0}(R)$ (resp. $D_{0}(R)$ ) (the Royden decomposition).

LEMMA 1.4. Every HD-function on $R$ has $\mu$-integrable boundary value on $\Delta$, where $\mu$ is the harmonic measure of $\Delta$ with respect to a point $z_{0} \in R$.

\section{Generalized harmonic measures.}

DEFINITION. A harmonic function $u$ on $R$ is called a generalized harmonic 
measure if the greatest harmonic minorant $u \wedge(1-u)$ of $u$ and $1-u$ vanishes identically on $R$ ([5]).

LEMMA 2.1 ([7]). Suppose that $u$ is a nonconstant generalized harmonic measure with finite Dirichlet integral on $R$. For each $0<r<1$, set $G_{r}=\{p \in R$; $u(p)>r\}$. Then

$$
(d u, \omega)_{R}=-\int_{\partial G_{r}} *_{\omega}
$$

for any $\omega \in \Gamma_{h}(R)$ with $\int_{\partial G_{r}}|\omega|<\infty$.

We note that $\int_{\partial G_{r}}|\omega|<\infty$ for almost all $r(0<r<1)$, where each relative boundary of an open set is oriented so that the open set lies on the lefthand side of the boundary (cf. [1], [9]).

Definition. We say that an exact differential $d u$ on $R$ belongs to the class $\Gamma \widehat{h m}(R)$ if there exists a sequence of functions $\left\{u_{n}\right\}$, each $u_{n}$ being a real linear combination of generalized harmonic measures with finite Dirichlet integral and $\left\|d u_{n}-d u\right\|_{R} \rightarrow 0(n \rightarrow \infty)$.

Then clearly $\Gamma_{h \mathrm{~m}}(R)$ is a closed subspace of $\Gamma_{h}(R)$.

\section{Partitions of the harmonic boundary.}

Definition. We say that $(P)=\left(P: \delta_{1}, \cdots, \delta_{N}\right)$ is a finite partition of the harmonic boundary $\Delta$ if $\delta_{1}, \cdots, \delta_{N}$ are mutually disjoint nonempty compact subsets of $\Delta$ and $\Delta=\delta_{1} \cup \cdots \cup \delta_{N}$.

DEFinition. An exact differential $d u$ in $\Gamma \widehat{h m}(R)$ belongs to the class $(P) \Gamma \widehat{h m}(R)$ if $u$ takes a constant value on each part $\delta_{j}(1 \leqq j \leqq N)$ of the partition $(P)$ of $\Delta$.

Proposition 3.1. The class $(P) \Gamma_{h m}(R)$ is a closed subspace in $\Gamma_{h m}(R)$.

Proof. Clearly $(P) \Gamma_{h m}(R) \subset \Gamma \widehat{h m}(R)$. Suppose that $d u_{n} \in(P) \Gamma_{h m}(R), d u \in$ $\Gamma_{h m}(R)$ and $\left\|d u_{n}-d u\right\|_{R} \rightarrow 0$. We may assume that there is a point $z_{0} \in R$ such that $u_{n}\left(z_{0}\right)=u\left(z_{0}\right)=0$ and $\left\{u_{n}\right\}$ converges to $u$ uniformly on every compact subset of $R$. Let $u_{n}=c_{n}^{(j)}$ on $\delta_{j}(1 \leqq j \leqq N)$.

First, we prove that $\left\{u_{n}\right\}$ is uniformly bounded. Suppose that $\left\{u_{n}\right\}$ is not uniformly bounded. We may assume that $c_{n}^{(1)} \leqq 0$ and $c_{n}^{(2)} \rightarrow \infty$. Let $M$ be an arbitraly positive number. Then for sufflciently large number $n, 0 \cup\left(u_{n} \cap M\right)=0$ on $\delta_{1},=M$ on $\delta_{2}$ and converges to $0 \cup(u \cap M)$ uniformly on every compact subset of $R$. Let $h$ be an $H B D$-function on $R$ such that $h=1$ on $\delta_{2}$ and $h=0$ on $\Delta-\delta_{2}$. Then $h\left(0 \cup\left(u_{n} \cap M\right)-M\right)$ converges to $h(0 \cup(u \cap M)-M)$ uniformly on every compact subset of $R$. Further, for sufficiently large number $n$, 


$$
\begin{aligned}
& h\left(0 \cup\left(u_{n} \cap M\right)-M\right) \in B D_{0}(R) \text { and } \\
& \qquad \begin{aligned}
&\left\|d\left(h\left(0 \cup\left(u_{n} \cap M\right)-M\right)\right)\right\|_{R} \leqq\left\|d\left(h u_{n}\right)\right\|_{R}+2 M\|d h\|_{R} \\
& \leqq 3\left(M\|d h\|_{R}+\|d u\|_{R}\right) .
\end{aligned}
\end{aligned}
$$

By Lemma 1.1, $h(0 \cup(u \cap M)-M) \in B D_{0}(R)$ and $M=u \cap M \leqq u$ on $\delta_{2}$. While, $H D$-function $u$ is $\mu$-integrable on $\Delta$ and $\mu\left(\delta_{2}\right)>0$, where $\mu$ is the harmonic measure with respect to $z_{0}$. This is a contradiction. Hence, $\left\{u_{n}\right\}$ must be uniformly bounded and $u \in H B D(R)$.

Since $\left\{c_{n}^{(j)}\right\}$ is uniformly bounded, we may assume that there are constants $c^{(1)}, \cdots, c^{(N)}$ such that $c_{n}^{(j)} \rightarrow c^{(j)}(n \rightarrow \infty)$ for each $j$. For each $\delta_{\jmath}$, let $g$ be an $H B D$-function on $R$ such that $g=1$ on $\delta$, and $g=0$ on $\Delta-\delta_{j}$. Then $g\left(u_{n}-c_{n}^{(j)}\right)$ $\in B D_{0}(R)$. By the similar argument above, we conclude that $g\left(u-c^{(j)}\right) \in B D_{0}(R)$. Hence $u=c^{(j)}$ on $\delta_{\jmath}$.

\section{Weakly dividing cycles.}

We say that $c$ is a curve on $R$ if $c$ is an image of a homeomorphic mapping from an open interval or the unit circle into $R$. Let $\left\{c_{k}\right\}$ be a set of (at most countable number of) oriented piecewise analytic curves clustering nowhere in $R$.

Let $(P)=\left(P: \delta_{1}, \cdots, \delta_{N}\right)$ be a finite partition of the harmonic boundary $\Delta$. We say that a formal sum $c=\Sigma c_{k}$ is a $(P)$-weakly dividing cycle in $R$ if there exists an open set $G$ such that

(1) $c=\sum c_{k}$ coincides with the relative boundary $\partial G$ of $G$,

(2) $\overline{\partial G} \cap \Delta=\varnothing$,

(3) for each $\delta_{j}$, it holds either $\delta_{j} \subset \bar{G} \cap \Delta$ or $\delta_{j} \subset \Delta-\bar{G}$,

where the closure is taken in $R^{*}$.

In (1), $\partial G$ is oriented so that $G$ lies on the left hand side of $\partial G$. So, if $G$ is the complement of a curve $\gamma$ in $R$, then $\partial G$ is the sum of two oriented curves $\gamma^{+}$and $\gamma^{-}$which have the same image as $\gamma$ and are oriented reversely. We write (1) simply $c=\partial G$. While, in (2) $\partial G$ is the topological relative boundary of $G$ in $R$.

We say that $c$ is a weakly dividing cycle if (1) and (2) hold ([7]).

We say that a property holds for almost every curve or almost all curves in a family of curves if the subfamily of exceptional curves has infinite extremal length (cf. [11]).

Definition. We say that a differential $\omega$ belongs to the class $(P) \Gamma_{h w e}(R)$ (resp. $\left.\Gamma_{h w e}(R)\right)$ if $\omega \in \Gamma_{h}(R)$ and $\int_{c} \omega=0$ for almost all $(P)$-weakly dividing cycles (resp. weakly dividing cycles) $c$.

We note that if $\omega \in \Gamma(R)$, then $\int_{c}|\omega|<\infty$ for almost every weakly dividing 
cycle and $(P)$-weakly dividing cycle $c$.

We know that the class $\Gamma_{h w e}(R)$ is a closed subspace in $\Gamma_{h}(R)$, and that the orthogonal decomposition

$$
\Gamma_{h}(R)=\Gamma_{h m}(R)+* \Gamma_{h w e}(R)
$$

holds ([7]). By the similar argument, we can prove that $(P) \Gamma_{h w e}(R)$ is a closed subspace of $\Gamma_{h}(R)$ and the following

Proposition 4.1. $\Gamma_{h}(R)=(P) \widehat{\Gamma_{h m}}(R)+*(P) \Gamma_{h w e}(R)$.

\section{Periods along the harmonic boundary.}

Let $V$ be a union of a finite number of regular subregions of $R$ with disjoint closures. By a regular region we mean one which is relatively compact and bounded by a finite number of disjoint analytic curves. Suppose that $R-\bar{V}$ is connected. Let $\Gamma_{x}$ be a subspace of $\Gamma_{h}$. We say that $\sigma \in \Gamma_{x}(R$ $-V)$ if $\sigma$ is a harmonic differential on a neighborhood of $(R-\bar{V}) \cup \partial V$ and $\sigma \in$ $\Gamma_{x}(R-\bar{V})$.

Let $(\delta, \Delta-\delta)$ be a partition of $\Delta$. For $\sigma \in \Gamma_{h}(R-V)$, we shall define the period of $\sigma$ along $\delta$.

LEMMA 5.1. Let $G$ be a subregıon of $R$ with piecewise analytic boundary. Let $\sigma$ be a harmonic differential on a nerghborhood of $G \cup \partial G$ such that $\in \Gamma_{h}(G)$ and $\int_{\partial G}|\sigma|<\infty$. If $\bar{G} \cap \Delta=\varnothing$, then $\int_{\partial G} \sigma=0$.

Proof. Let $\hat{G}$ be the double of $G$ along $\partial G$. If $\hat{G}$ is compact, then the statement clearly holds. Hence, we assume that $\hat{G}$ is noncompact. Since $\hat{G}$ has no Green's function, there exists an exhaustion $\left\{\Omega_{n}\right\}$ of $\hat{G}$ such that $\Omega_{n}$ is symmetric with respect to $\partial G$ and

$$
\lim _{n \rightarrow \infty} \int_{\partial \Omega_{n \cap G}}|\sigma|=0 \text {. }
$$

Since

$$
\int_{\partial \Omega_{n \cap G}} \sigma+\int_{\partial G_{\cap} \Omega_{n}} \sigma=0,
$$

we have $\int_{\partial G} \sigma=0$.

DEFINITION. Let $(\boldsymbol{\delta}, \Delta-\delta)$ be a partition of $\Delta$ and $v_{\delta}=v_{\delta}^{R-V}$ be an $H B D$. function on $R-V$ such that $v_{\delta}=1$ on $\delta$ and $v_{\delta}=0$ on $(\Delta-\delta) \cup \partial V$. We call $v_{\delta}$ a generalized harmonic measure of $\delta$ on $R-V$

Set $G_{r}=\left\{p \in R ; v_{\delta}(p)>r\right\}$ for $0<r<1$. Then $\partial G_{r}$ is a weakly dividing 
cycle such that $\bar{G}_{r} \cap \Delta=\delta$ and $G_{r} \subset R-V$. By Lemma 2.1, we have the following

Lemma 5.2. Let $\sigma \in \Gamma_{h}(R-V)$. Then

$$
\int_{\partial G_{r}} \sigma=\left(d v_{\delta}, * \sigma\right)_{R-V}
$$

for almost all $0<r<1$.

Definition. For $\sigma \in \Gamma_{h}(R-V)$, we define the period of $\sigma$ along $\delta$ as

$$
\int_{\delta} \sigma=-\int_{\partial G_{r}} \sigma=-\left(d v_{\delta}, * \sigma\right)_{R-V},
$$

where $r$ is the value for which Lemma 5.2 holds.

Proposition 5.3. Let $(\delta, \Delta-\delta)$ be a partition of $\Delta$ and $c=\partial G$ be a weakly dividing cycle such that $\bar{G} \cap \Delta=\delta$ and $G \subset R-V$. Let $\sigma \in \Gamma_{h}(R-V)$ with $\int_{c}|\sigma|<$ $\infty$. Then for almost all $0<r<1$,

$$
\int_{\partial G_{r}} \sigma=\int_{c} \sigma
$$

Proof. There is a $B D$-function $w$ such that $w=1$ on $\delta, w=0$ on $R-G$ and harmonic on $G$. Then the harmonic part of the Royden decomposition of $w$ on $R-\bar{V}$ is the generalized harmonic measure $v_{\delta}$ of $\delta$ on $R-V$. By Lemma 5.1 , for almost all $0<r<1$,

$$
\int_{c} \sigma=\int_{\partial(w>r)} \sigma=\int_{\partial G_{r}} \sigma .
$$

THEOREM 5.4. Let $(P)=\left(P: \delta_{1}, \cdots, \delta_{N}\right)$ be a partition of $\Delta$ and $\sigma \in \Gamma_{h}(R)$. Then $\sigma \in(P) \Gamma_{h w e}(R)$ if and only if $\int_{\delta_{j}} \sigma=0$ for all $\}$.

Proof. Let $v_{\jmath}=v_{\delta_{j}}^{R-V}$ be the generalized harmonic measure of $\delta$, on $R-V$, that is, $v_{j} \in H B D(R-V)$ such that $v_{j}=1$ on $\delta_{j}$ and $v_{j}=0$ on $\left(\Delta-\delta_{j}\right) \cup \partial V$. We extend $v_{\jmath}$ on $R$ so that $v_{j}=0$ on $V$. Let $v_{j}=w_{j}+p_{j}$ be the Royden decomposition of $v_{j}$ on $R$. Then $w$, is a generalized harmonic measure on $R$ such that $w_{\jmath}=1$ on $\delta_{j}$ and 0 on $\Delta-\delta_{j}$. Since $\left(d p_{j}, \sigma\right)_{R}=0$ for $\sigma \in \Gamma_{h}(R)$, we have

$$
\left(d w_{\jmath}, \sigma\right)_{R}=\left(d v_{\jmath}, \sigma\right)_{R-V} .
$$

Hence, $(P) \Gamma \widehat{h m}(R)$ being generated by $\left\{d w_{j}\right\}(1 \leqq j \leqq N)$ proves the assertion.

\section{6. (P) $\hat{L}_{1}$-operator.}

Let $V$ be a union of a finite number of relatively compact regular subregions of $R$ with disjoint closures. We assume that $R-\bar{V}$ is connected. 
THEOREM 6.1. Let $f \in C^{\omega}(\partial V)$ and $(P)=\left(P: \delta_{1}, \cdots, \delta_{N}\right)$ be a partition of the harmonic boundary $\Delta$. There exists a unique function $u \in H B D(R-V)$ satisfying the follwoing conditions:

$$
\begin{aligned}
& \left.u\right|_{\partial V}=f, \\
& u=\text { constant on } \delta_{\jmath}(1 \leqq j \leqq N), \\
& \int_{\delta_{j}} * d u=0(1 \leqq j \leqq N) .
\end{aligned}
$$

Proof. (Uniqueness) Suppose that $u_{1}, u_{2} \in H B D(R-V)$ satisfying (1), (2), (3). Then $u_{1}-u_{2}=0$ on $\partial V$ and $u_{1}-u_{2}$ is constant on each $\delta_{j}$. Let $v_{j}=v_{\delta_{j}}^{R-V}$ is a generalized harmonic measure of $\delta$, on $R-V$. Then (3) implies

$$
\int_{\delta_{\jmath}} * d\left(u_{1}-u_{2}\right)=\left(d v_{\jmath}, d\left(u_{1}-u_{2}\right)\right)_{R-V}=0 \quad(1 \leqq j \leqq N) .
$$

Since $u_{1}-u_{2}$ is a linear combination of $\left\{v_{j}\right\}$, we conclude that $u_{1}-u_{2} \equiv 0$ on $R-V$.

(Existence) The matrix whose $(i, j)$-element is defined by

$$
\int_{\delta_{i}} * d v_{j}=\left(d v_{i}, d v_{j}\right)_{R-V}
$$

is symmetric and positive definite. In fact, for real variables $x_{1}, \cdots, x_{N}$,

$$
\left\|\sum_{j=1}^{N} x_{j} d v_{j}\right\|_{R-V}^{2}=\sum_{\imath, j=1}^{N} x_{\imath} x_{j}\left(d v_{i}, d v_{j}\right)_{R-V} \geqq 0
$$

and the equality holds if and only if $\sum_{j=1}^{N} x_{j} d v_{j} \equiv 0$, i. e. $x_{\jmath}=0$ for all $j$.

Let $H f \in H B D(R-V)$ such that $H f=f$ on $\partial V$ and 0 on $\Delta$. Consider the function $u=H f+\sum_{j=1}^{N} c_{j} v$, where $c_{\jmath}$ are real constants. Then

$$
\sum_{j=1}^{N} c_{j} \int_{\delta_{i}} * d v_{\jmath}=\int_{\delta_{i}} * d u-\int_{\delta_{i}} * d H f
$$

There exist $c_{1}, \cdots, c_{N}$ such that $\int_{\delta_{i}} * d u=0(1 \leqq i \leqq N)$. Therefore, there exists $u$ satisfying (1), (2) and (3).

We denote the function $u$ in Theorem 6.1 by $(P) \hat{L}_{1} f$.

THEOREM 6.2. The operator $(P) \hat{L}_{1}$ from $C^{\omega}(\partial V)$ to $H B D(R-V)$ is a normal operator. That is, $(P) \hat{L}_{1}$ is a linear operator satisfying the following conditions:

$$
\begin{aligned}
& \left.(P) \hat{L}_{1} f\right|_{\partial V}=f, \\
& \min _{\partial V} f \leqq(P) \hat{L}_{1} f \leqq \max _{\hat{\boldsymbol{o}} V} f, \\
& \int_{\partial V} * d\left((P) \hat{L}_{1} f\right)=0 .
\end{aligned}
$$


NORMAL OPERATORS CONSTRUCTED FROM GENERALIZED HARMONIC MEASURES 261

Proof. It is easy to see that $(P) \hat{L}_{1}$ is a linear operator and satisfies (1).

We prove (2). Let $(P) \hat{L}_{1} f=c$, on $\delta_{\jmath}$. It is clear that $(P) \hat{L}_{1} 1=1$. Therefore, it is sufficient to see that if $f \geqq 0$ then all $c$, are non-negative. Suppose that there exists a $c_{j}<0$. We may assume that $c_{j}$ is the minimum value of $(P) \hat{L}_{1} f$ on $\Delta$. Let $\delta=\left\{p \in \Delta ;(P) \hat{L}_{1} f=c_{j}\right\}$. Then $\delta$ is a union of some parts of the partition $(P)$. For $\varepsilon>0$, let $G_{\varepsilon}=\left\{p \in R ;(P) \hat{L}_{1} f(p)<c_{j}+\varepsilon\right\}$. Then, for almost all sufficiently small $\varepsilon>0, \partial G_{\varepsilon}$ is a weakly dividing cycle such that $\bar{G}_{\varepsilon} \cap \Delta=\delta$, and $G_{\varepsilon} \subset R-V$ and

$$
\int_{\delta} * d\left((P) \hat{L}_{1} f\right)=-\int_{\partial G_{\varepsilon}} * d\left((P) \hat{L}_{1} f\right)<0 \text {. }
$$

Thus there exists a part $\delta_{k}$ of $(P)$ such that $\int_{\delta_{k}} * d\left((P) \hat{L}_{1} f\right)<0$. This contradicts the property (3) in Theorem 6.1.

Finally we prove (3). Let $v_{\jmath}=v_{\delta_{j}}^{R-V}$ be the generalized harmonic measure of $\delta$, on $R-V$. By the following Lemma, we have

$$
\begin{aligned}
\int_{\partial V} * d\left((P) \hat{L}_{1} f\right) & =-\left(d\left(1-\sum_{j=1}^{N} v_{\jmath}\right), d\left((P) \hat{L}_{1} f\right)\right)_{R-V} \\
& =\left(d\left(\sum_{j=1}^{N} v_{\jmath}\right), d\left((P) \hat{L}_{1} f\right)\right)_{R-V} \\
& =\sum_{j=1}^{N} \int_{\delta_{j}} * d\left((P) \hat{L}_{1} f\right)=0 .
\end{aligned}
$$

LEMma 6.3 ([7]). Suppose that $v \in H D(\mathrm{R}-V)$ and $v=0$ on $\Delta$. Then

$$
(d v, \omega)_{R-V}=-\int_{\partial V} v^{*} \omega
$$

for any $\omega \in \Gamma_{h}(R-V)$.

Proposition 6.4. For every $f \in C^{\omega}(\partial V)$,

$$
\left\|d\left((P) \hat{L}_{1} f\right)\right\|_{R-V}^{2}=-\int_{\partial V} f^{*} d\left((P) \hat{L}_{1} f\right) .
$$

Proof. We recall that $(P) \hat{L}_{1} f=H f+\sum_{j=1}^{N} c_{j} v_{j}$ in the proof of Theorem 6.1. Then by Lemma 6.3 ,

$$
\begin{aligned}
\left\|d\left((P) \hat{L}_{1} f\right)\right\|_{R-V}^{2} & =\left(d H f, d\left((P) \hat{L}_{1} f\right)\right)_{R-V}+\sum_{j=1}^{N} c_{\jmath}\left(d v_{\jmath}, d\left((P) \hat{L}_{1} f\right)\right)_{R-V} \\
& =-\int_{\partial V} H f^{*} d\left((P) \hat{L}_{1} f\right)+\sum_{j=1}^{N} c_{j} \int_{\delta_{j}} * d\left((P) \hat{L}_{1} f\right) \\
& =-\int_{\partial V} f * d\left((P) \hat{L}_{1} f\right) .
\end{aligned}
$$




\section{Refinement of partions.}

Definition. Let $(P)=\left(P: \delta_{1}, \cdots, \delta_{N}\right)$ and $\left(P^{\prime}\right)=\left(P^{\prime}: \delta_{1}^{\prime}, \cdots, \delta_{M}^{\prime}\right)$ are partitions of $\Delta$. We say that $\left(P^{\prime}\right)$ is a refinement of $(P)$ if each $\delta_{j}^{\prime}$ is a subset of some $\delta_{i}$.

LEMMA 7.1. If $\left(P^{\prime}\right)$ is a refinement of $(P)$ then

$$
\left\|d\left((P) \hat{L}_{1} f\right)\right\|_{R-V} \geqq\left\|d\left(\left(P^{\prime}\right) \hat{L}_{1} f\right)\right\|_{R-V}
$$

for every $f \in C^{\omega}(\partial V)$.

Proof. Let $u=(P) \hat{L}_{1} f$ and $u^{\prime}=\left(P^{\prime}\right) \hat{L}_{1} f$. Let $v_{j}^{\prime}=v_{\delta_{j}^{\prime}}^{R-V}$ be the generalized harmonic measure of $\delta_{j}^{\prime}$ on $R-V$. Then $u-u^{\prime}=\sum_{j=1}^{M} c_{j}^{\prime} v_{j}^{\prime}$, for some $c_{j}^{\prime}(1 \leqq j \leqq M)$ and

$$
\left(d\left(u-u^{\prime}\right), d u^{\prime}\right)_{R-V}=\sum_{j=1}^{M} c_{j}^{\prime} \int_{\delta_{j}^{\prime}} * d u^{\prime}=0
$$

Hence

and

$$
\left(d u, d u^{\prime}\right)_{R-V}=\left\|d u^{\prime}\right\|_{R-V}^{2}
$$

Thus

$$
0 \leqq\left\|d u-d u^{\prime}\right\|_{R-V}^{2}=\|d u\|_{R-V}^{2}-\left\|d u^{\prime}\right\|_{R-V}^{2} .
$$

$$
\|d u\|_{R-V} \geqq\left\|d u^{\prime}\right\|_{R-V} \text {. }
$$

8. $\hat{L}_{1}$-operator.

DEFINITION. We define a constant

$$
\kappa_{R-V}=\kappa_{R-V}(f)=\inf \left\{\left\|d\left((P) \hat{L}_{1} f\right)\right\|_{R-V}\right\},
$$

where the infimum is taken over all finite partitions $(P)$ of $\Delta$.

Since $\left\|d L_{0} f\right\|_{R-V} \leqq\|d v\|_{R-V}$ for any $v \in H B D(R-V)$ with $\left.v\right|_{\partial V}=f$, it follows that $\kappa_{R-V}>0$ for every non-constant function $f$ (see [12], [14], [15] for $L_{0^{-}}$ operator).

PROPOSITION 8.1. There exist a sequence of partitions $\left\{\left(P_{n}\right)\right\}$ of $\Delta$ and $u \in$ $H B D(R-V)$ such that

$$
\left(P_{n+1}\right) \text { is a refinement of }\left(P_{n}\right)(n=1,2, \cdots) \text {, }
$$

(2) $\quad\|d u\|_{R-V}=\kappa_{R-V}$,

$$
\left.u\right|_{\partial V}=f \text {, }
$$

$$
\left\|d\left(\left(P_{n}\right) \hat{L}_{1} f\right)-d u\right\|_{R-V} \longrightarrow 0 \quad(n \rightarrow \infty) .
$$


Proof. There is a sequence of partitions $\left(P_{n}\right)$ such that $\left\|d\left(P_{n}\right) \hat{L}_{1} f\right\|_{R-V} \rightarrow$ $\kappa_{R-V}(n \rightarrow \infty)$. By Lemma 7.1, we may assume that $\left(P_{n+1}\right)$ is a refinement of $\left(P_{n}\right)(n=1,2, \cdots)$. Let $u_{n}=\left(P_{n}\right) \hat{L}_{1} f$. By the same argument as in Lemma 7.1, for $n<m$,

$$
\left\|d u_{n}-d u_{m}\right\|_{R-V}^{2}=\left\|d u_{n}\right\|_{R-V}^{2}-\left\|d u_{m}\right\|_{R-V}^{2} .
$$

Hence, there exists a $u \in H B D(R-V)$ such that $\left.u\right|_{\partial V}=f,\left\|d u_{n}-d u\right\|_{R-V} \rightarrow 0(n \rightarrow$ $\infty)$ and $\|d u\|_{R-V}=\kappa_{R-V}$.

We note that $u$ does not depend on the choice of a sequence of partitions in Proposition 8.1. In fact, suppose that $\left\{\left(P_{n}^{\prime}\right)\right\}$ is another sequence of partitions such that $\left\|d\left(P_{n}^{\prime}\right) \hat{L}_{1} f\right\|_{R-V} \rightarrow \kappa_{R-V}(n \rightarrow \infty)$ and $\left(P_{n+1}^{\prime}\right)$ is a refinement of $\left(P_{n}^{\prime}\right)(n=$ $1,2, \cdots)$. Let $u_{n}^{\prime}=\left(P_{n}^{\prime}\right) \hat{L}_{1} f$ and $u^{\prime}=\lim _{n \rightarrow \infty} u_{n}^{\prime}$. There is a sequence of partitions $\left\{\left(P_{n}^{\prime \prime}\right)\right\}$ such that $\left(P_{n+1}^{\prime \prime}\right)$ is a refinement of $\left(P_{n}^{\prime \prime}\right)$ and $\left(P_{n}^{\prime \prime}\right)$ is a refinement of both $\left(P_{n}\right)$ and $\left(P_{n}^{\prime}\right)(n=1,2, \cdots)$. Let $u^{\prime \prime}=\lim _{n \rightarrow \infty} u_{n}^{\prime \prime}=\lim _{n \rightarrow \infty}\left(P_{n}^{\prime \prime}\right) \hat{L}_{1} f$. By the same argument as in Lemma 7.1, $\left(d\left(u_{n}^{\prime \prime}-u_{n}\right), d u_{n}^{\prime \prime}\right)_{R-V}=0$. Since $\left\|d u_{n}^{\prime \prime}-d u^{\prime \prime}\right\|_{R-V}$ $\rightarrow 0$ and $\left\|d u_{n}-d u\right\|_{R-V} \rightarrow 0$

$$
\begin{aligned}
\left(d u^{\prime \prime}, d u\right)_{R-V} & =\lim _{n \rightarrow \infty}\left(d u_{n}^{\prime \prime}, d u_{n}\right)_{R-V} \\
& =\lim _{n \rightarrow \infty}\left\|d u_{n}^{\prime \prime}\right\|_{R-V}^{2}=\left\|d u^{\prime \prime}\right\|_{R-V}^{2} .
\end{aligned}
$$

Hence

$$
0 \leqq\left\|d u^{\prime \prime}-d u\right\|_{R-V}^{2}=\|d u\|_{R-V}^{2}-\left\|d u^{\prime \prime}\right\|_{R-V}^{2}=0 .
$$

Thus, $u=u^{\prime \prime}$. Similarly, $u=u^{\prime}$.

For any $w \in H D(R)$, there exists a unique $H D$-function $I_{R-V}(w)$ on $R-V$ such that $I_{R-V}(w)=w$ on $\Delta$ and $I_{R-V}(w)=0$ on $\partial V$. We call $I_{R-V}(w)$ the inextremisation of $w$ to $R-V$. It is clear that $I_{R-V}$ is a linear operator.

LeMma 8.2 ([7]). If $u \in H D(R)$ with $d u \in \Gamma_{h m}(R)$ then $d I_{R-V}(u) \in \Gamma_{\widehat{h m}}(R-V)$.

THEOREM 8.3. For every $f \in C^{\omega}(\partial V)$, there exists a unique function $u \in$ $H B D(R-V)$ satisfying the following conditions.

$$
\left.u\right|_{\partial V}=f,
$$

(2) there exist a harmonic function $u_{h m}$ on $R$ with $d u_{h m} \in \Gamma \widehat{h m}(R)$ and $a$ Dirichlet potential $p$ on $R$ such that

on $R-V$ and

$$
u=u_{h m}+p
$$

$$
\int_{\delta}^{*} d u=0
$$

for any partition $(\boldsymbol{\delta}, \Delta-\delta)$ of $\Delta$ consisting of two parts. 
Proof. (Existence) We use the notation $u_{n}=\left(P_{n}\right) \hat{L}_{1} f$ and $u$ in Proposition 8.1 and its proof. We have already proved (1).

We prove (2). Let $H f \in H B D(R-V)$ such that $H f=f$ on $\partial V$ and $H f=0$ on $\Delta$. Since $u_{n}-H f=0$ on $\partial V, d\left(u_{n}-H f\right) \in \Gamma_{h m}(R-V)$. Hence $d(u-H f) \in$ $\Gamma_{h m}(R-V)$. We set $u_{n}-H f=0$ and $u-H f=0$ on $V$ so that $u_{n}-H f, u-H f \in$ $B D(R)$. The Royden decomposition gives $u_{n}-H f=w_{n}+q_{n}, u-H f=w+q$, where $w_{n}, w \in H B D(R)$ and $q_{n}, q \in B D_{0}(R)$. Since $d w_{n} \in \Gamma_{\hat{h m}}(R)$ and $\left\|d w_{n}-d w\right\|_{R} \rightarrow 0$, we have $d w \in \Gamma_{h m}(R)$. We can extend $H f$ to a $B D_{0}$-function on $R$ so that $u=w+(q+H f)$ on $R-V$. Denoting $w$ by $u_{h m}$ and $q+H f$ by $p$ gives (2).

Finally, we shall prove (3). Let $v_{\delta}=v_{\delta}^{R-V}$ be a generalized harmonic measure of $\delta$ on $R-V$. By the note following Proposition 8.1, we may assume that each $\left(P_{n}\right)$ is a refinement of $(\delta, \Delta-\delta)$. Then

$$
\int_{\delta}^{*} d u=\left(d u_{\delta}, d u\right)_{R-V}=\lim _{n \rightarrow \infty}\left(d v_{\delta}, d u_{n}\right)_{R-V}=0 .
$$

(Uniqueness) Let $u=u_{\hat{h m}}+p$ and $u^{\prime}=u_{\widehat{h m}}^{\prime}+p^{\prime}$ satisfy (1), (2) and (3). Then $u-u^{\prime}=I_{R-V}\left(u_{h m}-u_{\widehat{h m}}^{\prime}\right)$. By Lemma 8.2, $d\left(u-u^{\prime}\right) \in \Gamma_{\widehat{h m}}(R-V)$. There is a sequence $\left\{w_{n}\right\}$ of $H B D$-functions on $R-V$ each $w_{n}$ being a linear combination of generalized harmonic measures with finite Dirichlet integral on $R-V,\left.w_{n}\right|_{\partial V}$ $=0$ and $\left\|d w_{n}-d\left(u-u^{\prime}\right)\right\|_{R-V} \rightarrow 0$. While, by (3), we have $\left(d w_{n}, d\left(u-u^{\prime}\right)\right)_{R-V}=0$. Hence $d u-d u^{\prime} \equiv 0$.

We denote the function $u$ by $\hat{L}_{1} f$. Then $\left\|d \hat{L}_{1} f\right\|_{R-V}=\kappa_{R-V}(f)$.

THEOREM 8.4. The operator $\hat{L}_{1}$ from $C^{\omega}(\partial V)$ to $H B D(R-V)$ is a normal operator. That is, $\hat{L}_{1}$ is a linear operator satisfying the following conditions:

$$
\begin{aligned}
& \left.\hat{L}_{1} f\right|_{\partial V}=f, \\
& \min _{\partial V} f \leqq \hat{L}_{1} f \leqq \max _{\partial V} f, \\
& \int_{\partial V} * d \hat{L}_{1} f=0 .
\end{aligned}
$$

Proof. We use the notation $\left(P_{n}\right) \hat{L}_{1}$ in Proposition 8.1. By Theorem 6.2, $\left(P_{n}\right) \hat{L}_{1}$ are normal operators. By Proposition $8.1,\left(P_{n}\right) \hat{L}_{1} f$ converges to $\hat{L}_{1} f$ uniformly on every compact subset of $R-V$. Hence (1), (2) and (3) hold.

\section{An extremal property.}

For every $v \in H B D(R-V)$ there exists a unique $H B D$-function $E(v)$ on $R$ such that $E(v)=v$ on $\Delta$. We call $E(v)$ the extremisation of $v$ (see [7], [8]). It is clear that $E$ is a linear operator and satisfies the following 
NORMAL OPERATORS CONSTRUCTED FROM GENERALIZED HARMONIC MEASURES 265

LEMMA 9.1. Let $v \in H B D(R-V)$ and $v=w+p$ on $R-V$, where $w \in H B D(R)$ and $p \in B D_{0}(R)$. Then $E(v)=w$. Moreover, if $v=0$ on $\partial V$ then $I_{R-V}(E(v))=v$ on $R-V$.

THEOREM 9.2. Let $f \in C^{\omega}(\partial V)$. The function $\hat{L}_{1} f$ minimizes $\|d v\|_{R-V}$ in $v \in H B D(R-V)$ such that $\left.v\right|_{\partial V}=f$ and $d E(v) \in \Gamma_{\hat{h m}}(R)$.

Proof. Let $v \in H B D(R-V)$ such that $\left.v\right|_{\partial V}=f$ and $d E(v) \in \Gamma_{h m}(R)$. Since $d E(v)-d E\left(\hat{L}_{1} f\right) \in \Gamma_{h m}(R), d I_{R-V}\left(E(v)-E\left(\hat{L}_{1} f\right)\right)=d\left(v-\hat{L}_{1} f\right) \in \Gamma_{h m}(R-V)$ by Lemma 8.2. Hence there is a sequence $\left\{w_{n}\right\}$ of $H B D$-functions on $R-V$ such that each $w_{n}$ is a linear combination of generalized harmonic measures with finite Dirichlet integral, equals 0 on $\partial V$ and $\left\|d w_{n}-\left(d v-d \hat{L}_{1} f\right)\right\|_{R-V} \rightarrow 0$. Since $\left(d w_{n}, d \hat{L}_{1} f\right)_{R-V}=0,\left(d v, d \hat{L}_{1} f\right)_{R-V}=\left\|d \hat{L}_{1} f\right\|_{R-V}^{2}$. Hence, $\|d v\|_{R-V} \geqq\left\|d \hat{L}_{1} f\right\|_{R-V}$.

\section{Regular operators.}

An operator $L$ from $C^{\omega}(\partial V)$ to $H B D(R-V)$ is called a regular operator if

$$
\begin{gathered}
\left.L f\right|_{\partial V}=f, \\
(d L f, d L g)_{R-V}=-\int_{\partial V} f^{*} d L g
\end{gathered}
$$

for any $f, g \in C^{\omega}(\partial V)([17])$.

THEOREM 10.1. Let $(P)$ be a finite partition of $\Delta$. Then $(P) \hat{L}_{1}$ and $\hat{L}_{1}$ are regular operators.

Proof. It is sufficient to prove that $(P) \hat{L}_{1}$ satisfies (2). Let $H f \in H B D(R-$ $V)$ such that $H f=f$ on $\partial V$ and $H f=0$ on $\Delta$. Then $d\left((P) \hat{L}_{1} f-H f\right) \in \Gamma_{h m}(R-V)$. Hence

Thus

$$
\left(d\left((P) \hat{L}_{1} f-H f\right), \quad d\left((P) \hat{L}_{1} g\right)\right)_{R-V}=0 .
$$

$$
\begin{aligned}
\left(d\left((P) \hat{L}_{1} f\right), d\left((P) \hat{L}_{1} g\right)\right)_{R-V} & =\left(d H f, d\left((P) \hat{L}_{1} g\right)\right)_{R-V} \\
& =-\int_{\partial V} f^{*} d\left((P) \hat{L}_{1} g\right) .
\end{aligned}
$$

\section{Modulus functions.}

Let $V_{0}$ and $V_{1}$ be two relatively compact regular subregions of $R$ with disjoint closures. We assume that $R-\bar{V}_{0} \cup \bar{V}_{1}$ is connected. Let $f=0$ on $\partial V_{0}$ and $f=1$ on $\partial V_{1}$. Then $\int_{\partial V_{0}} * d \hat{L}_{1} f=\left\|d \hat{L}_{1} f\right\|_{R-V_{0} \cup V_{1}}^{2}>0$. Set $\hat{q}_{1}=\left(2 \pi / \int_{\partial V_{0}} * d \hat{L}_{1} f\right) \hat{L}_{1} f$.

THEOREM 11.1. There exists a unique $H B D$-function $\hat{q}_{1}$ on $R-V_{0} \cup V_{1}$ such that 


$$
\begin{aligned}
& \left.\hat{q}_{1}\right|_{\partial V_{0}}=0, \\
& \left.\hat{q}_{1}\right|_{\partial V_{1}}=\hat{k}_{1}=\text { constant, } \\
& \hat{L}_{1}\left(\left.\hat{q}_{1}\right|_{\partial V_{0} \cup \partial V_{1}}\right)=\hat{q}_{1} \quad \text { on } R-V_{0} \cup V_{1}, \\
& \int_{\partial V_{0}} * d \hat{q}_{1}=2 \pi .
\end{aligned}
$$

We call $\hat{q}_{1} \hat{L}_{1}$-modulus function on $R-V_{0} \cup V_{1}$ with respect to $\partial V_{0}$ and $\partial V_{1}$. The constant $e^{\hat{k}_{1}}$ is called the $\hat{L}_{1}$-modulus of $R-V_{0} \cup V_{1}$ with respect to $\partial V_{0}$ and $\partial V_{1}$.

We denote usual $L_{1}$-modulus function for $L_{1}$-operator by $q_{1}$ (see [15]). That is, $q_{1}$ satisfies (1), (2), (4) of Theorem 11.1 and $L_{1}\left(\left.q_{1}\right|_{\partial V_{0} \cup \partial V_{1}}\right)=q_{1}$ on $R$ $V_{0} \cup V_{1}$. If $\left.q_{1}\right|_{\partial V_{1}}=k_{1}, e^{k_{1}}$ is called the $L_{1}$-modulus of $R-V_{0} \cup V_{1}$ with respect to $\partial V_{0}$ and $\partial V_{1}$.

\section{An example.}

Now, we consider a two sheeted branched covering surface of the unit disk. Let $D$ be the unit disk and $\left\{a_{n}\right\},\left\{b_{n}\right\}$ be sequences of positive numbers such that $0<a_{0}<b_{0}<a_{1}<b_{1}<\cdots<a_{n}<b_{n}<\cdots$ and $\lim _{n \rightarrow \infty} a_{n}=\lim _{n \rightarrow \infty} b_{n}=1$. Consider the region obtained from $D$ by deleting the closed intervals $\left[a_{n}, b_{n}\right]$ ( $n=$ $0,1, \cdots)$. Join two such copies, one being $D_{0}$ and another being $D_{1}$, crosswise along $\left[a_{n}, b_{n}\right](n=0,1, \cdots)$, so as to obtain a 2 -sheeted branched covering surface $R$ of $D$. Denote by $\pi$ the projection from $R$ onto $D$. In [6], we show that the number of components of the harmonic boundary $\Delta$ of $R$ is at most 2. Moreover, if intervals $\left[a_{n}, b_{n}\right]$ are sufficiently small then $\Delta$ consists of two components and if gaps $\left(b_{n}, a_{n+1}\right)$ are sufficiently small then $\Delta$ is connected. (See $[6$, p. 639] for precise estimations.)

Let $U$ be the sufficiently small disk with center 0 in $D$ so that $\pi^{-1}(U)$ consists of two disjoint disk $V_{0}$ in $D_{0}$ and $V_{1}$ in $D_{1}$.

Denote by $\partial D_{k}$ the boundary of $D_{k}$ corresponding to $\{|z|=1\}-\{1\}(k=0,1)$. Note that every $H B D$-function $u$ on $R-V_{0} \cup V_{1}$ is uniquely determined by the boundary values on $\partial V_{0} \cup \partial V_{1} \cup \partial D_{0} \cup \partial D_{1}$. Moreover, if $d u \in \Gamma \widehat{h m}\left(R-V_{0} \cup V_{1}\right)$ then $u$ is constant on $\partial V_{0}, \partial V_{1}, \partial D_{0}$ and $\partial D_{1}$ respectively ([6]).

Let $\tau$ be the nontrivial covering transformation of $R$. Let $\phi$ be the anticonformal automorphism of $R$ which preserves the sheets $D_{0}, D_{1}$ and is identical with the mapping $z \mapsto \bar{z}$ on each sheet.

Let $f=0$ on $\partial V_{0}$ and 1 on $\partial V_{1}$. Since $R$ has one Stoïlow ideal boundary component, $L_{1}=(I) \hat{L}_{1}$ for the identity partition $(I)=(\Delta)$. Let $L_{1} f=k$ on $\Delta$. Since $\left(L_{1} f\right) \circ \tau=L_{1}(1-f)=1-L_{1} f, k=1-k$. Hence $k=1 / 2$.

Further, $\left(L_{1} f\right) \circ \psi \circ \tau=1-L_{1} f$. Hence $L_{1} f=1 / 2$ on $\cup_{n}\left[a_{n}, b_{n}\right]$.

If $\Delta$ is connected, then $L_{1} f=\hat{L}_{1} f$. If $\Delta$ is not connected, then $L_{1} f \neq \hat{L}_{1} f$. 
Proof. We shall prove the latter half. Contraly to the assertion, suppose that $L_{1} f=\hat{L}_{1} f$. Then $\hat{L}_{1} f=1 / 2$ on $\Delta$, hence on $\partial D_{0} \cup \partial D_{1}$, and $\hat{L}_{1} f=1 / 2$ on $\cup_{n}\left[a_{n}, b_{n}\right]$.

If $\Delta$ is not connected then there exists $v \in H B D\left(R-V_{0} \cup V_{1}\right)$ such that $v=0$ on $\partial V_{0} \cup \partial V_{1} \cup \partial D_{1}$ and $v=1$ on $\partial D_{0}$. Then $d v \in \Gamma_{h m}\left(R-V_{0} \cup V_{1}\right)$. Therefore, $\left(d v, d \hat{L}_{1} f\right)_{R-V}=0$. While,

$$
\begin{aligned}
\left(d v, d \hat{L}_{1} f\right)_{R-V} & =\left(d v, d\left(\hat{L}_{1} f-\frac{1}{2}\right)\right)_{R-V} \\
= & -\int_{\partial\left(V_{0} \cup V_{1}\right)}\left(\hat{L}_{1} f-\frac{1}{2}\right) * d v \\
= & \frac{1}{2}\left(\int_{\partial V_{0}} * d v-\int_{\partial V_{1}} * d v\right)>0 .
\end{aligned}
$$

For, $\left.v\right|_{D_{0}}-\left.v\right|_{D_{1}}$ is considered as an $H B D$-function on $D-U-\cup_{n}\left[a_{n}, b_{n}\right]$ whose boundary values equals 0 on $\left(\cup_{n}\left[a_{n}, b_{n}\right]\right) \cup \partial U$ and equals 1 on $\partial D-\{1\}$. This is a contradiction.

In the latter case, by Lemma 7.1 and its proof, we have $\left\|d L_{1} f\right\|_{R-V_{0} \cup V_{1}}>$ $\left\|d \hat{L}_{1} f\right\|_{R-V_{0} \cup V_{1}}$. Therefore, $\hat{k}_{1}>k_{1}$.

\section{REFERENCES}

[1] Accola, R., On semi-parabolic Riemann surfaces, Trans. Amer. Math. Soc., 108 (1963), 437-448.

[2] Ahlfors, L. ANd Sario, L., Riemann surfaces, Princeton Univ. Press (1960), 382 $\mathrm{pp}$.

[3] Constantinescu, C. And Cornea, A., Ideale Ränder Riemannscher Flächen, Springer-Verlag (1963), $244 \mathrm{pp}$.

[4] Fuglede, B., Extremal length and functional completion, Acta Math., 98 (1957), 171-219.

[5] Heins, M., On the Lindelöf principle, Ann. Math., 61 (1955), 440-473.

[6] IsHidA, H., Harmonic Dirichlet functions and the components of harmonic boundaries of Riemann surfaces, J. Math. Kyoto Univ., 29 (1989), 625-641.

[7] IsHidA, H., Weakly exact differentials on open Riemann surfaces, J. Math. Kyoto Univ., 30 (1990), 235-243.

[8] Kuramochi, Z., Relations between harmonic dimensions, Proc. Japan Acad., 30 (1954), 576-580.

[9] Marden, A. AND Rodin, B., Periods of differentials on open Riemann surfaces, Duke Math. J., 33 (1966), 103-108.

[10] NAKAI, M. AND SARIO, L., Construction of principal functions by orthogonal projection, Canad. J. Math., 18 (1966), 887-896.

[11] Ohtsuka, M., Dirichlet problem, extremal length and prime ends, Van Nostrand (1970), $326 \mathrm{pp}$.

[12] Rodin, B. and Sario, L., Principal functions, Van Nostrand (1968), 347 pp. 
[13] SARIO, L., A linear operator method on arbitraly Riemann surfaces, Trans. Amer. Math. Soc., 72 (1952), 281-295.

[14] SARIO, L. AND NAKaI, M., Classification theory of Riemann surfaces, SpringerVerlag (1970), $446 \mathrm{pp}$.

[15] SaRio, L. And Oikawa, K., Capacity functions, Springer-Verlag (1969), $361 \mathrm{pp}$.

[16] Watanabe, M., On a boundary property of principal functions, Pacific J. Math., 31 (1969), 537-545.

[17] Yamaguchi, H., Regular operators and spaces of harmonic functions with finite Dirichlet integral on open Riemann surfaces, J. Math. Kyoto Univ., 8 (1968), 169-198.

Department of Mathematics

KYOTO SANGYo UNIVERSITY 